\title{
Relaxor ferroelectric properties of 85PZN-15BT transparent ceramic at low temperatures
}

\author{
Lizhu Huang*,†, Wei Zhao*,†, Wei Ruan*, Heji Yang*, \\ Jiangtao Zeng* and Guorong Li*, \\ *Key Laboratory of Inorganic Functional Materials and Devices \\ Shanghai Institute of Ceramics, Chinese Academy of Sciences \\ Shanghai 200050, P. R. China \\ ${ }^{\dagger}$ Graduate School of Chinese Academy of Sciences \\ Beijing 100039, P. R. China \\ tgrli@mail.sic.ac.cn
}

Received 7 July 2013; Revised 14 August 2013; Accepted 27 August 2013; Published 11 October 2013

\begin{abstract}
Dielectric, hysteresis $(P-E)$ loops and TSDC properties of transparent $85 \mathrm{PZN}-15 \mathrm{BT}$ ceramic over $-150-150^{\circ} \mathrm{C}$ were investigated. The sample was found to exhibit a strong relaxor behavior and a relatively small remanent polarization. TSDC studies showed that there existed an electric field-dependent peak in the $p$ versus $T$. Such interesting features were attributed to the electric field-induced transition and coexistence of polar nanoregion, long-range ordered polar region and nonpolarizable BZN-rich region.

Keywords: PZN; BT; relaxor; electric field-induced transition; TSDC.
\end{abstract}

\section{Introduction}

Lead-based relaxor ferroelectric materials, known for their strong frequency dispersion of dielectric response, ${ }^{1-3}$ high dielectric constant, ${ }^{1}$ large electrostrictive coefficient ${ }^{4,5}$ and good transmittance, ${ }^{6}$ have been intensively studied over the past decades. It is commonly recognized that relaxors are highly inhomogeneous materials, which consist of randomly oriented polar nanoregions (PNRs) and chemically ordering regions (CORs). ${ }^{7}$ Moreover, the CORs block the PNRs growth to micrometer. An external electric field could overcome the energy barrier and transform the material into a long-range ordered polar state.

$\mathrm{Pb}\left(\mathrm{Zn}_{1 / 3} \mathrm{Nb}_{2 / 3}\right) \mathrm{O}_{3}$ (PZN) is a typical relaxor ferroelectrics. It undergoes a diffused phase transition above $140^{\circ} \mathrm{C}$. The perovskite structure PZN is thermal unstable and will turn to pyrochlore phase in the preparing process due to its inherently low tolerance factor and small absolute value of the bond valence sum of oxygen. ${ }^{8}$ To stabilize the perovskite structure, addition of a little amount of $\mathrm{BaTiO}_{3}$ (BT) is purposed as an effective way. ${ }^{9}$ Structurally, the nonpolarizable $\mathrm{BZN}$-rich region was found to exist in BT stabilized PZN by TEM analysis. ${ }^{10}$ However, the dynamical responses of PNR with strong electric field and temperature in such relaxor ferroelectrics are still less studied.

Here, we investigated the evolution of dielectric, hysteresis $(P-E)$ loops and domain with strong electric field and temperature of $85 \mathrm{PZN}-15 \mathrm{BT}$ transparent ceramic.

\section{Experiments}

Transparent $85 \mathrm{PZN}-15 \mathrm{BT}$ ceramic was fabricated by using a two-stage sintering method. The detailed preparation procedure can be found in our previous report. ${ }^{11}$ The sintered sample was polished to $0.2 \mathrm{~mm}$ and deposited with gold as electrodes for different property measurements. Dielectric properties were measured by using a Novocontrol Broadband Dielectric Spectrometer with a $500 \mathrm{~V}$ DC high voltage booster. Thermally simulated depolarization current (TSDC) properties were measured by using a Keithley 6517 Electrometer. Ferroelectric hysteresis $(P-E)$ loops and curves of current versus electric field were characterized by using a TF2000 using the Sawyer-Tower circuit at $10 \mathrm{~Hz}$. Temperature with a precision of $0.01 \mathrm{~K}$ was controlled by using a Novocontrol Quatro Cryosystem.

\section{Results and Discussion}

\subsection{Dielectric properties under DC bias}

Figure 1 shows dielectric property as a function of temperature for the $85 \mathrm{PZN}-15 \mathrm{BT}$ ceramic under various conditions (ZFH: zero-field-heating, FC: field-heating under DC bias after ZFH). The broad maximum and frequency dispersion of dielectric permittivity indicates its relaxor-like behavior. With increasing DC bias, a drop of dielectric permittivity within a wide temperature range and a shift of $T_{\mathrm{m}}$ can be observed. Such features reveal an electricfield-induced transition from relaxor state to a long-range ordered ferroelectric phase or a mixed state phase as reported in many previous works. Threshold field $E_{\mathrm{th}}$ has also been observed by measuring dielectric permittivity (at $1 \mathrm{kHz}$ ) of the sample that has been prepoled under DC field within $1 \mathrm{~min}$ at room temperature, as shown in the insert of Fig. 2. Anomalous drop in dielectric permittivity near $5 \mathrm{kV} / \mathrm{cm}$ was observed, indicating the presence of $E_{\mathrm{th}}$ for the $85 \mathrm{PZN}-15 \mathrm{BT}$ ceramic. 


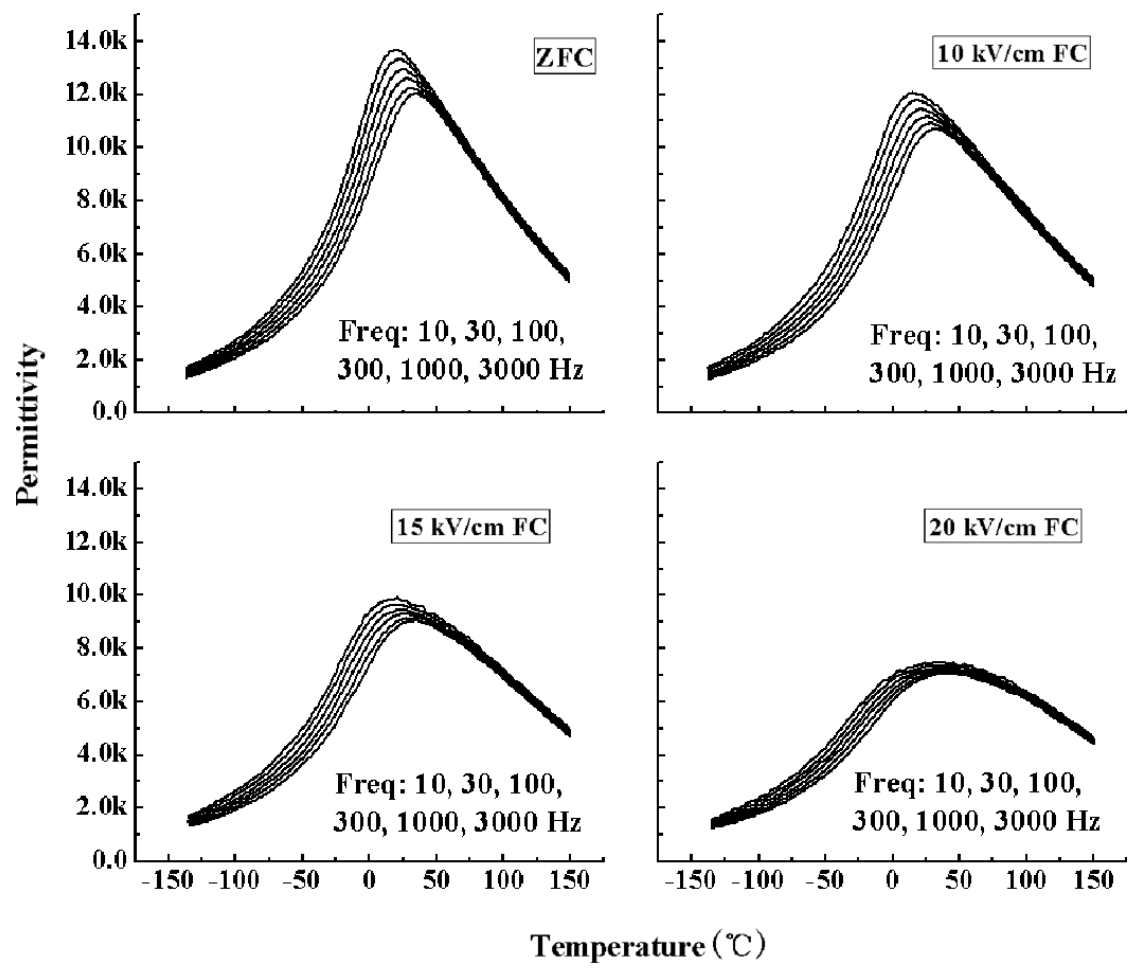

Fig. 1. Dielectric property as a function of temperature and DC bias for the transparent 85PZN-15BT ceramic.

There is no obvious shrink of dielectric permittivity, even under high electric field of $20 \mathrm{kV} / \mathrm{cm}$. Such strong relaxor feature indicates that certain amount of PNRs still exist at measured lowest temperature. Generally, PNRs are not stable and easily grow under electric field at low temperatures; as a consequence, the permittivity is expected to shrink at low temperatures under electric field, which is commonly less than $10 \mathrm{kV} / \mathrm{cm}$. For example, dielectric permittivity of $4 \%$ La-doped 75PMN-25PT shrinks below electric field of $4 \mathrm{kV} / \mathrm{cm} .{ }^{12}$ One main mechanism suggests to enhance local random fields derived from the existence of nonpolarizable $\mathrm{BZN}$-rich region.

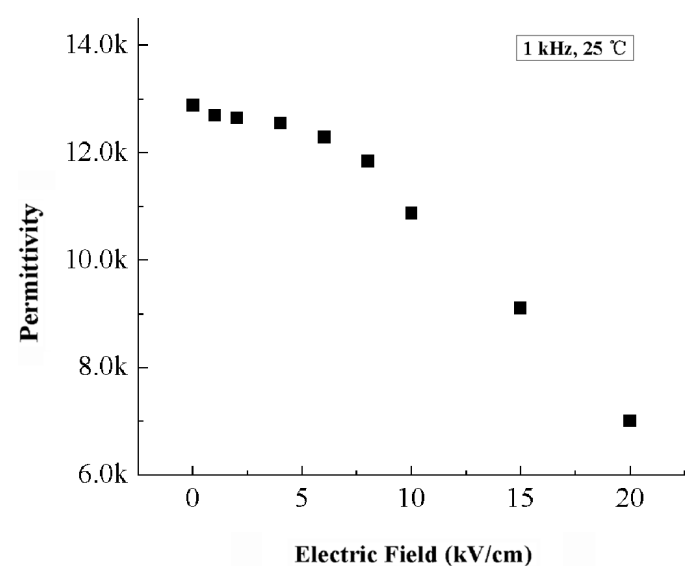

Fig. 2. Dielectric permittivity at $1 \mathrm{kHz}$ as a function of applied DC field at room temperature.

\subsection{Ferroelectric properties}

Figure 3 shows hysteresis $(P-E)$ loops at various temperatures, ranging from $-150^{\circ} \mathrm{C}$ to $140^{\circ} \mathrm{C}$. Clearly, the evolution of $P-E$ loops gives a direct evidence that there are paraelectricrelaxor and relaxor-normal transition. Below the freezing temperature of $-75^{\circ} \mathrm{C}$ calculated using Vogel-Fulcher model, or in the temperature from relxaor to normal ferroelectric phase

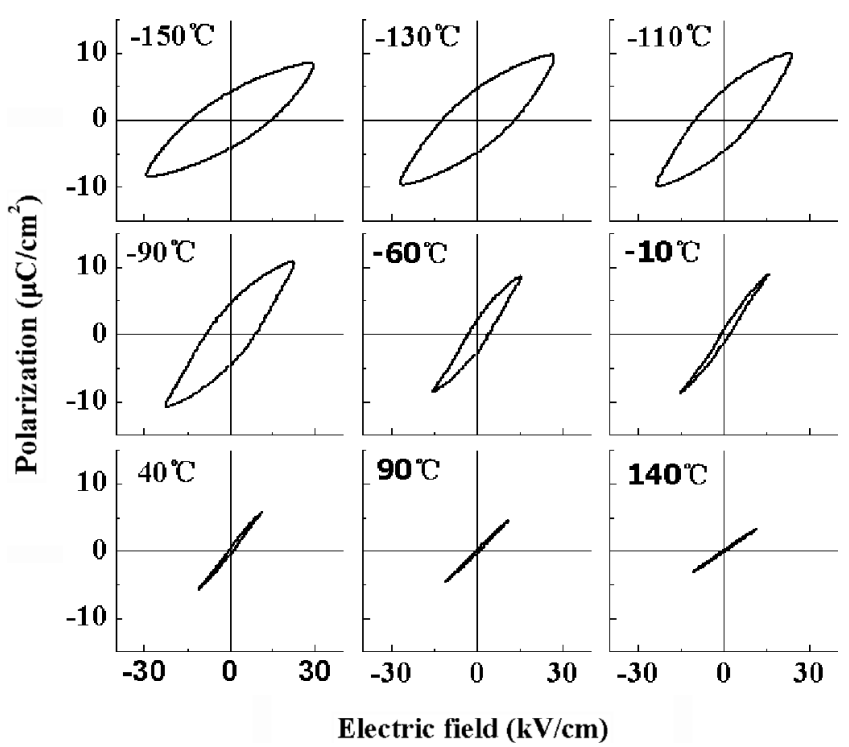

Fig. 3. Evolution of ferroelectric properties of the sample at $10 \mathrm{~Hz}$ with temperature. 
transition in our previous report, ${ }^{11}$ one can see that the 85PZN-15BT ceramic has square hysteresis loop which is a characteristic of typical normal ferroelectrics, indicating the existence of long-range ordered polarization derived from electric field and temperature. The saturated and remanent polarizations of the $85 \mathrm{PZN}-15 \mathrm{BT}$ are very small with comparison to that of typical ferroelectrics. Such phenomenon can be explained by the coexistence of polar nanoregion, longrange ordered polar region and nonpolarizable BZN-rich region. This is also supported by dielectric analysis.

\subsection{TSDC properties}

To better investigate dynamical evolution of PNRs under electric fields and thermal stability of long-range ordered polar state, TSDC was measured, as shown in Fig. 4. The samples were heated to $100^{\circ} \mathrm{C}$, polarized at various DC electric fields, and rapidly cooled to $-150^{\circ} \mathrm{C}$ while keeping DC fields. The samples were stayed at $-150^{\circ} \mathrm{C}$ for $5 \mathrm{~min}$ after removing the DC fields, then were heated to $70^{\circ} \mathrm{C}$ at $3^{\circ} \mathrm{C} / \mathrm{min}$ while measuring the current. Similar to what have been reported by Cross et al., ${ }^{13}$ the sample has very small magnitude of maximum current $I_{\max }$, relatively not too high remanent polarization and a broad peak. It is well known that the peak in the current versus $T$ represents the transition from long-range ordered domain to microdomain state. Obviously, such welldefined broad peak can be observed for all electric fields. However, while increasing electric field, the current peak increases and shifts to lower temperature until reaching saturation state at $15 \mathrm{kV} / \mathrm{cm}$. The peak temperature shift indicates that there is no structure phase transition in the temperature range of $-150 \sim-50^{\circ} \mathrm{C}$, and high current at high electric fields reveals that higher electric field could induce larger macrodomain state, but larger macrodomains are more unstable because the peaks of $I_{\max }$ shift to lower temperature.

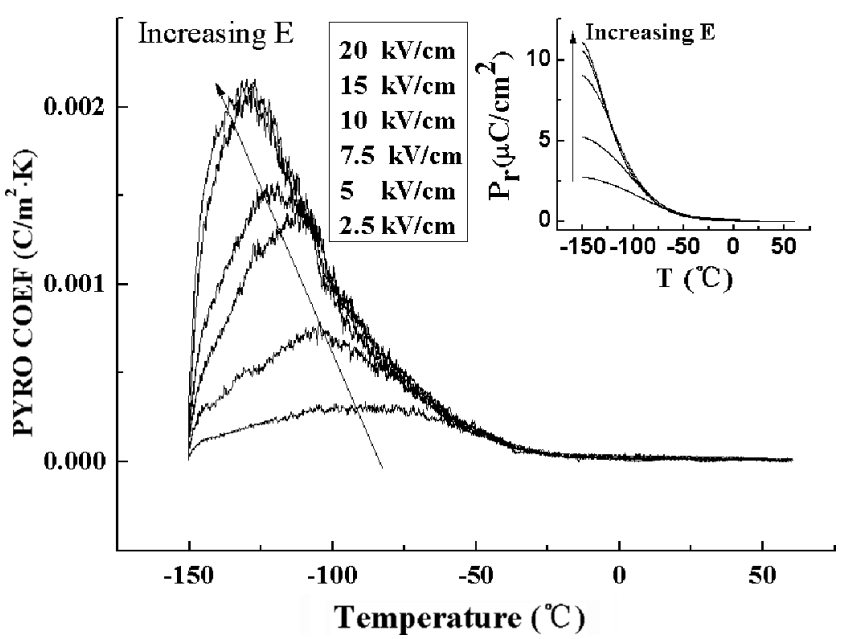

Fig. 4. TSDC properties of $85 \mathrm{PZN}-15 \mathrm{BT}$ as a function of temperature cooled under various electric fields. Insert is calculated polarization versus temperature.
The calculated remanent polarization is shown in the insert of Fig. 2. Small remanent polarization was obtained, which coincides with what observed from ferroelectric measurement.

\subsection{Discussion}

Dielectric, hysteresis $(P-E)$ loops and TSDC properties suggested that $85 \mathrm{PZN}-15 \mathrm{BT}$ ceramic has an electric fieldinduced transition from relaxor state to a long-range ordered ferroelectric phase or a mixed state phase. The polar nanoregions appeared at high temperatures beyond the temperature of maximum dielectric constant versus temperature, $T_{\mathrm{m}}$, and then formed nuclei for the field-induced long-range order at low temperatures. In such transition, the competition, as well as coexistence between short-range and long-range polar order, were dominated by local random fields. For BT stabilized PZN, it was suggested that there existed nonpolarizable BZNrich regions. Such regions resulted in high local random fields which enhanced the relaxor characteristics or, in other words, increased the energy barrier that would inhibit the fieldinduced phase transition. Thus, there appeared strong relaxor behavior, quite broad TSDC peak and relatively small remanent polarization in $85 \mathrm{PZN}-15 \mathrm{BT}$ transparent ceramic. At measured lowest temperatures in the present work, it could be expected that there was a coexistence of polar nanoregion, long-range ordered polar region and nonpolarizable BZNrich region.

\section{Conclusion}

In summary, we studied dielectric, ferroelectric and pyroelectric properties of transparent $85 \mathrm{PZN}-15 \mathrm{BT}$ ceramic. A clear feature of polar nanoregions and electric field-induced long-range order was drawn. Nonpolarizable BZN-rich region was suggested to exist and enhance local random fields. The existence of nonpolarizable region resulted in strong relaxor behavior, quite broad pyroelectric peak and relatively small remanent polarization.

\section{Acknowledgments}

This work was supported by the National Key Project for Basic Research of China (2009CB623305), the National Nature Science Foundation of China (Nos. 61137004, 51107140, and 61275181), and the External Cooperation Program of the Chinese Academy of Sciences (No. GJHZ1042).

\section{References}

${ }^{1}$ L. E. Cross, Relaxor ferroelectrics, Ferroelectrics 76, 241 (1987).

${ }^{2}$ A. A. Bokov and Z. G. Ye, Dielectric relaxation in relaxor ferroelectrics, J. Adv. Dielect. 2, 1241010 (2012).

${ }^{3}$ V. V. Westphal, W. Kleemann and M. D. Glinchuk, Diffuse phase transitions and random-field-induced domain states of the "relaxor" ferroelectric $\mathrm{PbMg}_{1 / 3} \mathrm{Nb}_{2 / 3} \mathrm{O}_{3}$, Phys. Rev. Lett. 68, 847 (1992). 
${ }^{4}$ S. E. Park and T. R. Shrout, Relaxor based ferroelectric single crystals for electro-mechanical actuators, Mater. Res. Innovat. 1, 20 (1997).

${ }^{5}$ S. E. Park and T. R. Shrout, Ultrahigh strain and piezoelectric behavior in relaxor based ferroelectric single crystals, J. Appl. Phys. 82, 1804 (1997).

${ }^{6}$ A. Sternberg, Transparent ferroelectric ceramics - recent trends and status QUO, Ferroelectrics 131, 13 (1992).

${ }^{7} \mathrm{~W}$. Kleemann, Random fields in relaxor ferroelectrics - a jubilee review, J. Adv. Dielect. 2, 1241001 (2012).

${ }^{8}$ N. Wakiya, N. Ishizawa, K. Shinozaki and N. Mizutani, Thermal stability of $\mathrm{Pb}\left(\mathrm{Zn}_{1 / 3} \mathrm{Nb}_{2 / 3}\right) \mathrm{O}_{3}(\mathrm{PZN})$ and consideration of stabilization conditions of perovskite type compounds, Mater. Res. Bull. 30, 1121 (1995).

${ }^{9}$ A. Halliyal, U. Kumar, R. E. Newnham and L. E. Cross, Dielectric and ferroelectric properties of ceramics in the $\mathrm{Pb}\left(\mathrm{Zn}_{1 / 3} \mathrm{Nb}_{2 / 3}\right) \mathrm{O}_{3}-$ $\mathrm{BaTiO}_{3}-\mathrm{PbTiO}_{3}$ system, J. Am. Ceram. Soc. 70, 119 (1987).
${ }^{10}$ S. Y. Chen, S. Y. Cheng and C. M. Wang, Effect of Barium Titanate on microstructural evaluation and properties of Lead Zinc Magnesium Niohatcceramics, J. Am. Ceram. Soc. 74, 400 (1991).

${ }^{11}$ L. Z. Huang, J. T. Zeng, W. Ruan, W. Zhao, K. Y. Zhao and G. R. $\mathrm{Li}$, Fabrication and dielectric properties of transparent PZN-BT ceramic, Ceram. Int. 39, s171 (2013).

${ }^{12}$ W. Ruan, G. R. Li, J. T. Zeng and L. S. Kamzina, Origin of the giant electro-optic Kerr effect in La-doped 75PMN-25PT transparent ceramics, J. Appl. Phys. 110, 074109 (2011).

${ }^{13}$ U. Kumar and L. E. Cross, Pyroelectric and electrostrictive properties of $(1-x-y) \mathrm{PZN} \cdot \mathrm{xBT} \cdot \mathrm{yPT}$ ceramic solid solutions, J. Am. Ceram. Soc. 75, 2155 (1992). 